\title{
Global and local dynamical invariants and quasienergy states of time-periodic Hamiltonians
}

\author{
D. B. Monteoliva ${ }^{1}$, B. Mirbach ${ }^{2 *}$, and H. J. Korsch ${ }^{3}$ \\ ${ }^{1}$ Departamento de Física, Universidad Nacional de La Plata, C.C.6\%, 1900 La Plata, Argentina \\ ${ }^{2}$ Facolta die Science, Universitá di Milano, sede di Como, I-22100 Como, Italy \\ ${ }^{3}$ Fachbereich Physik, Universität Kaiserslautern, D-67653 Kaiserslautern, Germany \\ (e-mail: korsch@physik.uni-kl.de) \\ (July 4, 1997)
}

\begin{abstract}
A formalism is developed for calculating the quasienergy states and spectrum for time-periodic quantum systems when a time-periodic dynamical invariant operator with a nondegenerate spectrum is known. The method, which circumvents the integration of the Schrödinger equation, is applied to an integrable class of systems, where the global invariant operator is constructed. Furthermore, a local integrable approximation for more general non-integrable systems is developed. Numerical results are presented for the doubleresonance model.
\end{abstract}

PACS number(s): 03.65.-w, 05.45.+b

\section{INTRODUCTION}

The time dependent Schrödinger equation can be integrated directly [1], or approximated, e.g. by analytic Dyson or Magnus [2-4] expansions to obtain the evolution of the system in the form of the fundamental propagator. For time periodic systems, the Floquet theory $[5,6]$ provides a unique functional form for a propagator, which permits to construct the solution for any time from the first cycle. Using the periodic form of the Floquet propagator, the problem can be reformulated in terms of solutions (quasienergy states) and eigenvalues (quasienergies) which characterize and determine the dynamics of the system. Nevertheless, the Schrödinger equation has still to be integrated over the first cycle.

We know from classical mechanics that an integrable system, when perturbed, leads to soft chaos in the sense of the KAM theorem [7]. That is, the phase space of the integrable system is entirely stratified by invariant tori, specified by actions $I_{j}$, on which the motion is linear in time and characterized by the frequencies $\omega_{j}=\omega_{j}\left(I_{j}\right)$. The neighborhood of the resonance tori corresponding to commensurable frequencies with rational winding numbers $\Omega=\omega_{i} / \omega_{j}=r / s$ (integer $r, s$ ) are usually destroyed in the presence of perturbations. For the remaining tori,

*present address: MPI für Physik komplexer Systeme, D01167 Dresden the EBK quantization $[7,8]$ allows a semiclassical approximation of the quasienergy spectrum.

We present here an entirely quantum mechanical formalism, which allows us to construct the quasienergy spectrum and quasienergy states from the knowledge of a global invariant operator of the system. In this way, the integration of the Schrödinger equation, often a laborious task, can be avoided.

It is well known that generic time-periodic Hamiltonians are non-integrable and both regular and chaotic motions coexist $[9,10]$. Here we discuss systems, where the chaotic component can still be considered as small and the main characteristics are dominated by regular dynamics. For an approximate treatment of such systems, two different strategies can be employed: First, one can make use of the semiclassical EBK quantization to quantize the states supported by classical invariant tori [8] (by means of interpolation, this method can also be used to quantize weak chaotic components $[11,12])$. Alternatively, one can construct an integrable approximation to the Hamiltonian, which can then be treated quantum mechanically in (almost) closed form.

In Sect. II, the general formalism is developed and, in the following two sections, applied to integrable systems and non-integrable ones for which approximate (local) invariant operators are constructed. First, in Sect. III, a class of integrable systems is presented and its invariant operator form is derived. Then the formalism is applied to a specific example: a single resonance system. In Sect. IV, the non-integrable double-resonance model is treated as an example where an approximate invariant operator can be constructed. Approximate quasienergies and quasienergy states are computed and compared with exact results and a previously suggested harmonic oscillator approximation. Finally, in Sect. V, we summarize our results.

\section{FORMALISM}

A quantum system described by a time-periodic Hamiltonian (period $T$ ) evolves from an initial state $|\Psi(0)\rangle$ to the state $|\Psi(t)\rangle=\hat{U}(t)|\Psi(0)\rangle$ according to the Schrödinger equation 


$$
\mathrm{i} \hbar \dot{\hat{U}}(t)=\hat{H}(t) \hat{U}(t),
$$

where $\hat{U}(t)$ is the unitary evolution operator with the initial condition $\hat{U}(0)=\hat{1}$. As $\hat{H}(t)$ is $T$-periodic, Floquet theory $[5,6]$ provides a fundamental set of solutions: the Floquet or quasienergy states

$$
\left|\Psi_{\alpha}(t)\right\rangle=\hat{U}(t)\left|\Psi_{\alpha}(0)\right\rangle=\mathrm{e}^{-\mathrm{i} \varepsilon_{\alpha} t / \hbar}\left|\phi_{\alpha}(t)\right\rangle,
$$

where $\left|\phi_{\alpha}(t+T)\right\rangle=\left|\phi_{\alpha}(t)\right\rangle$ and $\varepsilon_{\alpha}$ is the quasienergy. The quasienergy states are obtained as the eigenvalues and eigenvectors of the Floquet operator - the time evolution operator $\hat{U}(T)$ over one period $T$ of the system:

$$
\hat{U}(T)\left|\Psi_{\alpha}(0)\right\rangle=\mathrm{e}^{-i \varepsilon_{\alpha} T / \hbar}\left|\Psi_{\alpha}(0)\right\rangle .
$$

Let us now assume that the system possesses a hermitian invariant operator $\hat{I}(t)=\hat{I}^{\dagger}(t)$,

$$
\frac{\mathrm{d} \hat{I}(t)}{\mathrm{d} t}=\frac{\partial \hat{I}(t)}{\partial t}+\frac{1}{\mathrm{i} \hbar}[\hat{I}(t), \hat{H}(t)] \equiv 0,
$$

which is one of a complete set of commuting observables, so that there is a complete set of eigenstates of $\hat{I}(t)$. Furthermore, $\hat{I}(t)$ should not involve time-derivative operators. Then, following Lewis and Riesenfeld [13], the general solution of the Schrödinger equation is

$$
|\Psi(t)\rangle=\sum_{\lambda, k} c_{\lambda k}\left|\psi_{\lambda k}(t)\right\rangle,\left|\psi_{\lambda k}(t)\right\rangle=\mathrm{e}^{i \alpha_{\lambda k}(t)}|\lambda, k ; t\rangle
$$

where $|\lambda, k ; t\rangle$ are the normalized instantaneous eigenstates of $\hat{I}(t)$ with eigenvalues $\lambda$. The index $k$ describes the degeneracy of the eigenvalue $\lambda$ and the $c_{\lambda k}$ are $t$ independent. We call the particular state $\left|\psi_{\lambda k}(t)\right\rangle$ a Lewis and Riesenfeld $\lambda k$ solution. The Lewis phases $\alpha_{\lambda k}(t)$ are determined by the expectation value of the Floquet operator $\mathrm{i} \hbar \partial / \partial t-\hat{H}(t)$ with respect to the eigenstates of the invariant:

$$
\hbar \dot{\alpha}_{\lambda k}(t)=\left\langle\lambda, k ; t\left|\mathrm{i} \hbar \frac{\partial}{\partial t}-\hat{H}(t)\right| \lambda, k ; t\right\rangle,
$$

provided that the $\left\langle\lambda, k^{\prime} ; t|\mathrm{i} \hbar \partial / \partial t-\hat{H}(t)| \lambda, k ; t\right\rangle$ are zero for $k^{\prime} \neq k$ (this is always possible, because the Floquet operator $\mathrm{i} \hbar \partial / \partial t-\hat{H}(t)$ is hermitian and is therefore diagonalizable).

In addition, we assume that the invariant operator is $T$-periodic and its eigenvalues are non-degenerate: $|\lambda, k ; t\rangle=,|\lambda ; t\rangle$. In this case, they are also $T$-periodic $|\lambda ; T\rangle=|\lambda ; 0\rangle$, and a particular $\lambda$ solution $\left|\psi_{\lambda}(t)\right\rangle$ of $(5)$ after one period is

$$
\begin{aligned}
\hat{U}(T)\left|\psi_{\lambda}(0)\right\rangle=\left|\psi_{\lambda}(T)\right\rangle & =\mathrm{e}^{\mathrm{i} \alpha_{\lambda}(T)}|\lambda ; 0\rangle \\
& =\mathrm{e}^{\mathrm{i} \alpha_{\lambda}(T)}\left|\psi_{\lambda}(0)\right\rangle .
\end{aligned}
$$

Comparing (3) with (7), the quasienergies are identified as

$$
\begin{aligned}
\varepsilon_{\lambda} & =-\frac{\hbar}{T} \alpha_{n}(T) \bmod \hbar \omega \\
& =-\frac{1}{T} \int_{0}^{T}\left\langle\lambda ; t\left|i \hbar \frac{\partial}{\partial t}-\hat{H}(t)\right| \lambda ; t\right\rangle \mathrm{d} t \bmod \hbar \omega
\end{aligned}
$$

up to a factor of $\hbar \omega=2 \pi \hbar / T$. Rewriting $\left|\psi_{\lambda}(t)\right\rangle$ as

$$
\begin{aligned}
\left|\psi_{\lambda}(t)\right\rangle & =\mathrm{e}^{\mathrm{i} \alpha_{\lambda}(T) t / T}\left|\phi_{\lambda}(t)\right\rangle \\
\left|\phi_{\lambda}(t)\right\rangle & =\mathrm{e}^{\mathrm{i}\left(\alpha_{\lambda}(t)-\alpha_{\lambda}(T) t / T\right)}|\lambda ; t\rangle,
\end{aligned}
$$

where $\left|\phi_{\lambda}(t)\right\rangle$ is the $T$-periodic state vector in (2), because of $\alpha_{\lambda}(t+T)=\alpha_{\lambda}(t)+\alpha_{\lambda}(T)$, and $|\lambda ; t\rangle$ is $T$ periodic. It is clear that the Lewis and Riesenfeld solutions $\left|\psi_{\lambda}(t)\right\rangle$ are the quasienergy states.

As mentioned in the introduction, there is no need here to know the evolution operator $\hat{U}(t)$, whose calculation is frequently a difficult task. If a $T$-periodic invariant operator $\hat{I}(t)$ not involving $t$-derivatives can be found with a complete set of non-degenerate eigenstates $|\lambda ; t\rangle$, the quasienergy spectrum can be determined from (8), being in one-to-one relation with the spectrum of the invariant, while (9) and (10) determine the quasienergy states.

In a previous article $[14,15]$, we have shown that it is in principle possible to find such an invariant operator for systems whose Hamiltonian is generated by a dynamical algebra closed with respect to the action of the commutator. We have treated there two examples of such integrable systems, the generalized harmonic oscillator and the two-level system.

In the following section, we apply the formalism to a class of integrable systems, constructing first its invariant operator and then considering a single resonance system as a specific example.

\section{AN INTEGRABLE CASE}

Examples of integrable systems are rare, but because of their conceptual importance they are discussed repeatedly in the literature. By far the most intensively analyzed system is the celebrated time-dependent harmonic oscillator, where the importance of the dynamical Lewis invariant is well established (see, e.g., [14] and references given there). Here we discuss a very simple example of an integrable system with Hamiltonian form

$$
\hat{H}=\hat{H}(\hat{x}-\omega t, \hat{p})
$$

with $[\hat{x}, \hat{p}]=\mathrm{i} \hbar$ ( $\omega$ is a real number). Transforming into a rotating frame

$$
\hat{x}^{\prime}=\hat{x}-\omega t \quad, \quad t^{\prime}=t
$$

the Schrödinger equation can be written as

$$
\mathrm{i} \hbar \frac{\partial}{\partial t}\left|\Psi\left(\hat{x}^{\prime}, t\right)\right\rangle=\left(\hat{H}\left(\hat{x}^{\prime}, \hat{p}^{\prime}\right)-\omega \hat{p}^{\prime}\right)\left|\Psi\left(\hat{x}^{\prime}, t\right)\right\rangle .
$$


with $\hat{p}^{\prime}=-\mathrm{i} \hbar \partial / \partial x^{\prime}=\hat{p}$. The operator $\hat{H}\left(\hat{x}^{\prime}, \hat{p}^{\prime}\right)-\omega \hat{p}^{\prime}$ does not depend explicitly on time in this frame and is therefore a constant of motion. Thus, going back to the former frame, this constant of motion will define an invariant operator for the system defined by the Hamiltonian (11):

$$
\hat{I}(\hat{x}-\omega t, \hat{p})=\hat{H}(\hat{x}-\omega t, \hat{p})-\omega \hat{p}
$$

as can be easily checked by inserting (14) into (4).

For this class of systems, regardless on the particular form of the $\hat{p}$ or $\hat{x}$ dependence as far as time-dependence goes together with $\hat{x}$ as in (11), an invariant operator of the form (14) exist and the Lewis and Riesenfeld phase can be found from (6) as

$$
\alpha_{\lambda k}(t)=-\frac{\lambda}{\hbar} t
$$

where $\lambda$ is the (time independent) eigenvalue of the invariant (14).

As a particular time-periodic example of the class of systems defined by (14), we consider the Hamiltonian

$$
\hat{H}=-\frac{\hbar^{2}}{2} \frac{\partial^{2}}{\partial \varphi^{2}}-\frac{f}{2} \cos (\varphi-\omega t)
$$

which models, e.g., the planar motion of a dipole (a diatomic molecule in the rigid rotor approximation) in a time-periodic electric field with circular polarization. The invariant operator is given by (14) (the frequency $\omega$ of the field can be taken to be unity by rescaling the time). It should be noted that for one-dimensional systems with quadratic $\hat{p}$-dependence, a general dynamical invariant has been drived by Ray [16]. The dynamical invariant operator (14) with Hamiltonian (16) is in complete agreement with this result up to an irrelevant constant term $\omega^{2} / 2$, which is evident by using $F(t)=w(t)=k=0, \rho(t)=1$ and $G=-f / 2 \cos (x-\omega t)$ in eqns. (1.5) to (1.8) of [16].

To construct the quasienergy states, we solve the eigenvalue equation for the invariant operator

$$
\hat{I}(t)|\lambda ; t\rangle=\lambda|\lambda ; t\rangle
$$

which leads to the Mathieu equation

$$
\ddot{u}+(a-2 q \cos 2 y) u=0,
$$

where the dots denote derivation with respect to $y=$ $(\varphi-t+\pi) / 2$, and the parameter $q$ is given by

$$
q=2 f / \hbar^{2} .
$$

The characteristic values $a$ determine the eigenvalues $\lambda$ of the invariant

$$
\lambda=\frac{1}{2}\left(\frac{\hbar^{2} a}{4}-1\right)
$$

and the wave functions are given by

$$
\langle\varphi \mid \lambda ; t\rangle=\mathrm{e}^{i(\varphi-t) / \hbar} u\left(\frac{\varphi-t+\pi}{2}\right) .
$$

The $2 \pi$-periodic boundary conditions for the eigenstates (21) yield $\pi$-periodic solutions of the Mathieu equation. Now, the $\pi$-periodic solutions correspond to the countable infinite set of characteristic values $c_{2 n}(q), n=0,1, \ldots[19]$. For convenience, they are often separated in two sets: $a_{2 n}(q)$, which yield even $\pi$-periodic solutions $u_{2 n}^{\mathrm{e}}(y)$, and $b_{2 n}(q)$, which yield odd $\pi$-periodic solutions $u_{2 n}^{\mathrm{o}}(y)$. For fixed real $q>0$ the characteristic values $a_{2 n}$ and $b_{2 n}$ are real and distinct (therefore they are non-degenerate) and ordered as $a_{0}<b_{2}<a_{2}<b_{4}<$ ... Therefore, the eigenstates $|\lambda ; t\rangle$ of the invariant satisfying the boundary conditions are

$$
\left\langle\varphi \mid \lambda_{n} ; t\right\rangle=\mathrm{e}^{i(\varphi-t) / \hbar} u_{2 n}\left(\frac{\varphi-t+\pi}{2}\right) .
$$

As for this system the invariant operator satisfies the conditions discussed above, the quasiangles $\theta_{n}=\varepsilon_{n} T / \hbar$, from (8) and (15), are

$$
\theta_{n}=\frac{2 \pi \lambda_{n}}{\hbar} \bmod 2 \pi=\frac{\pi}{\hbar}\left(\frac{\hbar^{2} c_{2 n}}{4}-1\right) \bmod 2 \pi
$$

determined by the characteristic values $c_{2 n}(q)$ up to multiples of $2 \pi$. The quasienergy states are therefore (see (9) and $(10))$

$$
\left|\psi_{n}(t)\right\rangle=\mathrm{e}^{-\mathrm{i}\left(\hbar^{2} c_{2 n} / 4-1\right) t / 2 \hbar}\left|\lambda_{n}, t\right\rangle .
$$

As demonstrated in the following section, an integrable system of this type can be used to construct local approximations to the quasienergy states of non-integrable systems. In this way, the spectral structure of the system under consideration can be analyzed.

\section{A NON-INTEGRABLE CASE}

As mentioned in the introduction, global nonintegrability of a system seems to be the rule. However, for classical systems we know that some local approximate invariants may exist as can be easily seen by a simple inspection of the Poincaré surface of section. Moreover, Chirikov showed that, within a so-called secular perturbation theory, dynamics in the vicinity of nonlinear resonances can be described by the (integrable) motion of a generalized pendulum [20,9]. It would be instructive to construct approximations for these invariants in quantum mechanics. In this section, we discuss the problem for a typical example, the double-resonance model.

In dimensionless units, the Hamiltonian is

$$
\hat{H}=-\frac{\hbar^{2}}{2} \frac{\partial^{2}}{\partial \varphi^{2}}-f \cos t \cos \varphi,
$$


which, as before, represents, e.g., a rigid planar diatomic molecule interacting with an electro-magnetic field whose direction is now fixed in space, which makes the system non-integrable. This system is also known as the doubleresonance model as is clearly seen by rewriting the Hamiltonian as

$$
\hat{H}=-\frac{\hbar^{2}}{2} \frac{\partial^{2}}{\partial \varphi^{2}}-\frac{f}{2}[\cos (\varphi-t)+\cos (\varphi+t)] .
$$

Because of its fundamental importance, this model has been studied by several authors [21-28,30] also because of applications to collisional and spectroscopic experiments, where molecules are oriented in an electric field [29].

For the classical system, two fundamental resonances with frequencies $\Omega\left(J_{+}\right)=1$ and $\Omega\left(J_{-}\right)=-1$ at $J_{ \pm}=$ \pm 1 are immediately recognized, where the system rotates clockwise or anti-clockwise with the field. The centers of these main resonances appear as stable fixed points in the stroboscopic Poincaré section shown in Fig. 1 for a scaled field strength $f=0.1$, where a few classical trajectories are shown in momentum space at times $t=n T, n=$ $1,2, \ldots$.

\section{$\mathrm{J}$}

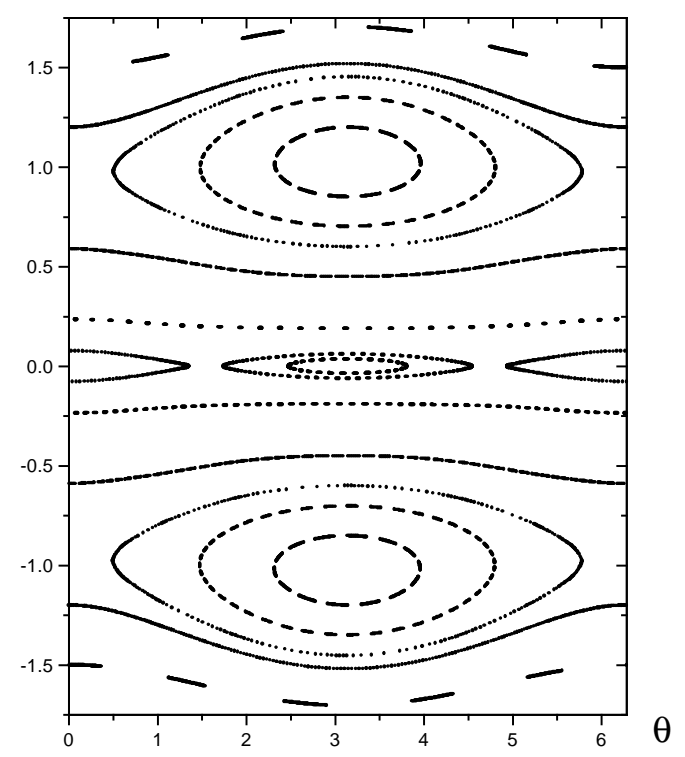

FIG. 1. Classical stroboscopic Poincaré section for a driven rotor with force amplitude $f=0.1$ at times $t=n T, n=1,2, \ldots$, where $T=2 \pi$ is the period of the field $(\Theta=\varphi+\pi)$. Two primary resonances with winding numbers $\Omega\left(J_{+}\right)=1$ and $\Omega\left(J_{-}\right)=-1$ are clearly seen. As a result of the perturbation between them, a secondary resonance coupling the motion of the two primary islands can also be observed.

For small values of the parameter $f$, the width of the resonances is small compared to their distance leaving the system almost integrable. As a result of the perturbation between the two resonances, invariant tori around the separatrix of each resonance are deformed. With increasing parameter $f$ more and more tori are destroyed and the Poincaré section shows a (bounded) extended chaotic zone generated by a single classical trajectory [26]. In Fig. 1, the resonance layer is still very thin and can only be seen as a thickening of the separatrix. A secondary resonance coupling the motion between the two primary islands is also observed.

In a quantum treatment of this system, we can make an attempt to construct a local invariant operator for the individual resonances. Around each primary resonance the system has a slowly varying term (see (26)) and a rapidly varying one which, after averaging, yields a negligible contribution [9,28]. Thus, we approximate the system by the co- or counter-rotating Hamiltonian

$$
\hat{H}^{ \pm}=-\frac{\hbar^{2}}{2} \frac{\partial^{2}}{\partial \varphi^{2}}-\frac{f}{2} \cos (\varphi \mp t),
$$

respectively. The $\hat{H}^{ \pm}$are integrable, with an associated invariant operator (14):

$$
\hat{I}^{ \pm}=\hat{H}^{ \pm} \pm \mathrm{i} \hbar \frac{\partial}{\partial \varphi} .
$$

Thus, following the formalism of Section II, the approximate quasienergy spectrum is given by (23) and its quasienergy states $\left|\psi_{n}(t)\right\rangle$ by (24) with

$$
\left\langle\varphi \mid \lambda^{ \pm}, t\right\rangle=\exp [ \pm i(\varphi \pm t / \hbar)] u_{2 n}((\varphi \mp t \pm \pi) / 2) .
$$

Note that the quasienergies are identical for the two cases.

In order to explore the validity of this simple approximation, we solve the Schrödinger equation numerically for the exact Hamiltonian given by (26) and compare the results. The the evolution operator (3) is propagated to $t=T$ by means of the $\left(t, t^{\prime}\right)$-method [1]. For this purpose, the quasienergy states (2) are expanded in the free-rotor basis

$$
\left|\Psi_{\alpha}(0)\right\rangle=\sum_{m} C_{m, \alpha}|m\rangle
$$

and then the coefficients $C_{m, \alpha}$ are determined numerically. The free-rotor Hamiltonian $\hat{H}_{0}=\hat{J}^{2} / 2$ is in the following used to label the states by $\alpha=1,2,3, \ldots$ in increasing order of its expectation value

$$
\begin{aligned}
\left\langle\hat{H}_{0}\right\rangle_{\alpha} & =\left\langle\Psi_{\alpha}(0)\left|\frac{\hat{J}^{2}}{2}\right| \Psi_{\alpha}(0)\right\rangle \\
& =\frac{\hbar^{2}}{2} \sum_{m=-\infty}^{\infty} m^{2}\left|C_{m, \alpha}\right|^{2},
\end{aligned}
$$

i.e. the kinetic energy.

Because of the symmetry of the Hamiltonian, the quasienergy states will have a definite parity. Even 
quasienergy states are obtained when the free-rotor states $|m\rangle$ in the expansion (30) are

$$
\left\langle\varphi_{\mathrm{e}} \mid 0\right\rangle=\frac{1}{\sqrt{2 \pi}} ; \quad\left\langle\varphi_{\mathrm{e}} \mid m\right\rangle=\frac{1}{\sqrt{\pi}} \cos (m \varphi)
$$

and odd ones, when the $|m\rangle$ are

$$
\left\langle\varphi_{\mathrm{o}} \mid m\right\rangle=\frac{1}{\sqrt{\pi}} \sin (m \varphi)
$$

for $m=1,2, \ldots$.

In the numerical calculations, we use a value of $\hbar=$ 0.01 , which yields converged results when the expansion (30) is confined to $|m| \leq 200$. In order to identify the quasienergy states which localize on the resonances, one can construct and analyze the (Husimi) phase space distribution of each quasienergy state. A much simpler method [27] is to plot the expectation value of the kinetic energy (31) against the entropy

$$
S_{\alpha}=-\sum_{m}\left|C_{m, \alpha}\right|^{2} \ln \left|C_{m, \alpha}\right|^{2} .
$$

of each state as a measure of the degree of delocalization of the state $\alpha$ in the free-rotor basis. Such an energyentropy plot is shown in Fig. 2 for the even quasienergy states (a similar diagram is obtained for the odd states). We observe a clear distinction between three types of quasienergy states. The upper branch corresponds to states $\left|\Psi_{\alpha}(0)\right\rangle$ associated with the outer regular region above the upper and below the lower resonance in Fig. 1. The horizontal branch is made up of the states associated with the regular regions inside the fundamental resonances and the lower branch corresponds to states associated with the region between the primary resonances. In addition, there is a second horizontal branch with approximately zero kinetic energy, which is related to the states localized on the inner resonance chain.

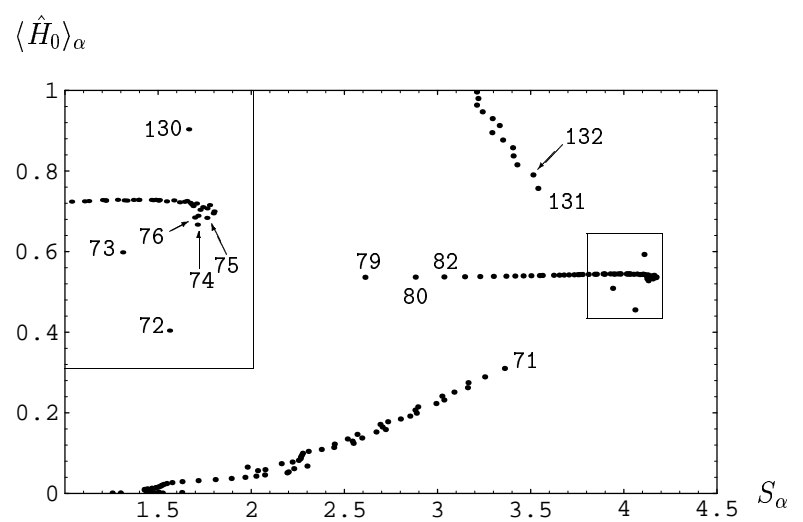

FIG. 2. Expectation value of the free rotor Hamiltonian with respect to the even quasienergy states $\left\langle\hat{H}_{0}\right\rangle_{\alpha}$ against its corresponding entropy $S_{\alpha}$. A few values of $\alpha$ are explicitly given. Three groups of states are distinguished. The inset shows a magnification of the boxed part of the spectrum, where distorted separatrix states are clearly seen.
Let us discuss the primary resonance states in some more detail. The states on the horizontal branch $\left\langle H_{0}\right\rangle_{\alpha} \approx 0.5$ with small entropy $S_{\alpha}$ correspond to the localized inner states of the resonances. The state with the smallest entropy, $\alpha=79$, localizes on the centers of the upper and lower resonance as can be verified by inspection of the Husimi distributions. With increasing entropy $S_{\alpha}$, the states populate wider phase space regions inside the resonance and the most highly delocalized states with large values of $S_{\alpha}$, are the ones near the separatrix of the primary resonances.

For increasing field strength, the coupling between the resonances is stronger and points on the lower and upper branch and the ones on the horizontal branch with high values of $S_{\alpha}$ develop into on a chaotic region. A 'chaotic cloud' of states with high entropy and almost the same average kinetic energy appears [26,30]. The beginning of this development is already seen in Fig. 2, where some states do not really belong to the upper and lower branches, but are distorted separatrix states.

As a semiclassical estimate, the number of states inside the fundamental resonance is calculated from the area inside the resonance divided by $2 \pi \hbar$, which leads to a number of 57 states inside each resonance for $f=0.1$.

The horizontal branch in Fig. 2 consists of 59 even states with $\alpha$ varying from 72 to 130 . By simple inspection, one sees that the outer separatrix states are the ones from $\alpha=72$ up to $\alpha=130$ for the even states and $\alpha=71$ to 129 for the odd ones. The even states with $\alpha=73,74,75$ and 76, and the odd ones with $\alpha=72,73,74$ and 75 belong to the resonance but they are close to the separatrix and therefore considerably perturbed.

The states localized at the resonance should be ordered, as far as the resonance approximation works, by the expectation value of the invariant operator $\hat{I}$ of the single-resonance system (compare Sect. III), i.e.

$$
\begin{aligned}
\langle\hat{I}\rangle_{\alpha} & =\left\langle\Psi_{\alpha}(0)|\hat{I}| \Psi_{\alpha}(0)\right\rangle=\frac{\hbar^{2}}{2} \sum_{m=1}^{\infty} m^{2}\left|C_{m, \alpha}\right|^{2} \\
& -\frac{f}{2} \sum_{m=1}^{\infty} \operatorname{Re}\left(C_{m, \alpha} C_{m+1, \alpha}^{*}\right)-\frac{f}{\sqrt{2}} \operatorname{Re}\left(C_{0, \alpha} C_{1, \alpha}^{*}\right) .
\end{aligned}
$$

The lowest value of $\langle\hat{I}\rangle_{\alpha}$ should correspond to the innermost resonance state, the next to the second, and so on, as illustrated in Fig. 3. The states 74 and 76 do not follow the ordering with $\langle\hat{I}\rangle_{\alpha}$. The states with $\alpha=72$ and 130 are completely out of the scale of this plot confirming the above criteria about the strongly perturbed states. They all belong to the 'chaotic cloud'.

We are now in position to make a comparison with the quasienergy spectrum of the resonance states of the integrable system of Sect. III. For the invariant approximation the ordering is clear: the first corresponds to the lowest value of $c_{2 n}, a_{0}$, the second to $b_{2}$, and so on. For 
the non-integrable system, we exclude the states with $\alpha=73,74$ and 76 which can not be ordered in this way. The results are shown in Table I, where the exact numerical quasiangles, $\theta_{\alpha}^{\mathrm{ex}}$, are compared with the approximation $(23), \theta_{\alpha}^{\mathrm{I}}$. (The characteristic values $c_{2 n}$ were calculated from the roots of continuous fractions $[19$, eqs. $(20.2 .13),(20.2 .21),(20.2 .23)]$.

The horizontal line in Table I marks the semiclassical position of the separatrix for the integrable case. As one can observe, the agreement is surprisingly good in view of the crude approximation in terms of the invariant of the single-resonance case. Also shown in the table is the harmonic oscillator approximation $\theta_{\alpha}^{\text {ho }}$ developed in [27]. This approximation describes the lowest resonance states and breaks down already for the $7^{\text {th }}$ state, whereas the present one works quite well up to the $54^{\text {th }}$ state. In particular, the approximation works well also for states above the separatrix.

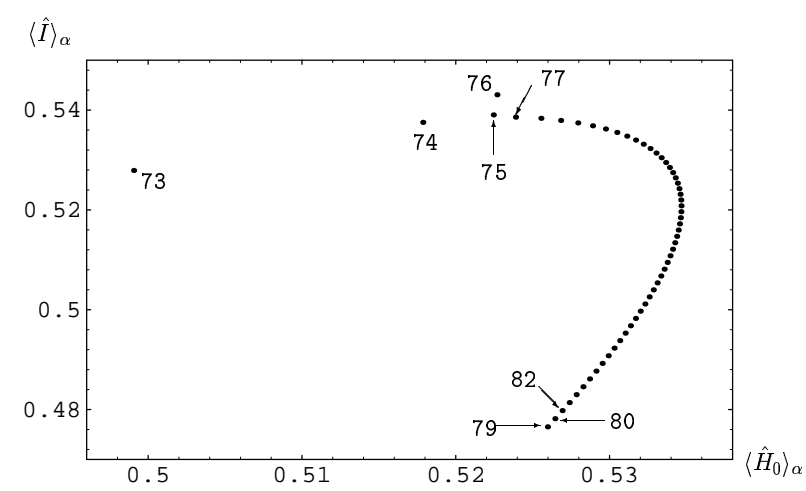

FIG. 3. Expectation value of the local invariant with respect to the even quasienergy states $\langle\hat{I}\rangle_{\alpha}$ against the expectation value of the free-rotor Hamiltonian $\left\langle\hat{H}_{0}\right\rangle_{\alpha}$ for the same states.

There is, however, a systematic deviation of about 0.1 for all the resonance states. This offset of the approximated quasiangles can be attributed to the deformation of the trajectory of the stable fixed point at the center of the resonance because of the perturbation by the second one.

Quantitatively, this can be taken into account by a (semi-)classical consideration: In [8] it has been discussed in a general context in which way the resonant dynamics can be mapped onto the integrable motion described by the Chirikov-Hamiltonian $H=E_{0}+\frac{G}{2} p^{2}-\frac{F}{2} \cos (\varphi-$ $\frac{r}{s} \omega t$ ) (compare (16)) by using data directly drawn from the classical dynamics. It was shown that the three parameters of this Hamiltonian are determined by the area of the resonance as well as the rotational frequency $\omega_{\text {p.o. }}$ and the action $S_{\text {p.o. }}$ of the central elliptic orbit, where the latter determines the constant energy shift $E_{0}$ (for more details see [8]; note, however, that the definition of $E_{0}$ given there differs slightly from the present one).
Assuming the other two parameters to be determined correctly in (27) (i.e. $G=1$ and $F=f$ ), one easily finds the constant energy shift to be

$$
E_{0}=-S_{\text {p.o. }} / 2 \pi+1 / 2+f / 2 .
$$

Consequently, expression (20) adopts the generalized form

$$
\lambda=-\frac{S_{\text {p.o. }}}{2 \pi}+\frac{1}{2}\left(\frac{\hbar^{2} a}{4}+f\right) .
$$

Using the expansion $c_{2 n}=-2 q+2 \sqrt{q}(2 n+1)+\mathcal{O}(1)$ of the characteristic values of the Mathieu-equation [19], one obtains for the low-lying states the harmonic oscillator approximation

$$
\lambda_{n}=-\frac{S_{\text {p.o. }}}{2 \pi}+\hbar \omega_{\text {p.o. }}\left(n+\frac{1}{2}\right),
$$

where $\omega_{\text {p.o. }}=\sqrt{f / 2}$ is the frequency of rotation around the elliptic orbit. For an alternative derivation of (38), based on a canonical transformation to a coordinate frame relative to the periodic orbit, see [12].

In a numerical simulation of the classical dynamics, the action of the elliptic orbit was determined as $S_{\text {p.o. }}=3.45475$, yielding a global shift in the quasiangles of $\theta_{0}=2 \pi E_{0} / \hbar=0.1005$. The semiclassical values, corrected by this shift, are listed as $\theta_{\alpha}^{\text {II }}$ in Table I. They show for all states inside the separatrix an excellent agreement with the exact numerical results. For the states located outside the separatrix (listed in Table I below the horizontal line) the agreement is gradually declining. For states in-between the two resonances $(\alpha \leq 72)$ this is not unexpected, since the approximation of the dynamics by one resonance only cannot work perfectly there. One the other hand, for very large energy the system approaches the free rotor, such that for very large $\alpha$ the (non-corrected) approximate values $\theta_{\alpha}^{\text {I }}$ will slowly converge towards the exact ones. This convergence is, however, not visible within the range of presented results.

\section{CONCLUSIONS}

We have presented here a formalism for calculating the quasienergy spectrum and states of $T$-periodic systems when a $T$-periodic global invariant operator is known. Although the association between the invariants and the quasienergies is already clear for semiclassical quantization theories, it appears here in a purely quantum context and is definitely more direct: there is a one-to-one relation between the spectrum and eigenstates of the invariant and the quasienergy spectrum and quasienergy states. An example, the integrable single-resonance model, has been studied in detail showing clearly this correspondence. 
Furthermore, the formalism was applied to a nonintegrable system where some local invariants can be approximated: the double-resonance model. It is however made clear that the problem is here shifted to the search of good invariant operator approximations, a problem that for time-dependent quantum systems should be studied with more detail.

An interesting problem arises here: when a nonintegrable system can be solved by the approximation of several local invariants, one may be tempted to ask whether it is possible to calculate their mutual perturbation in a way similar to the one usually applied in the case of double-well potentials. For the rigid-rotor presented here, the invariants were approximated locally around each resonance, yielding a double-degenerate quasienergy spectrum. It seems to be possible - by an invariant interaction analysis - to calculate the shifts and splittings of the quasienergies. Work along this line is in progress and results will appear elsewhere.

\section{ACKNOWLEDGMENTS}

This work has been supported by the Deutsche Forschungsgemeinschaft (SPP 'Zeitabhängige Phänomene und Methoden in Quantensystemen der Physik und Chemie'). DBM is grateful to the Graduiertenkolleg 'Laser- und Teilchen-Spektroskopie' and to the Universität Kaiserslautern for financial support.

[1] U. Peskin and N. Moiseyev, J. Chem. Phys. 99 (1993) 4590.

[2] W. Magnus, Commun. Pure Appl. Math. 7 (1954) 649.

[3] P. Pechukas and J. Light, J. Chem. Phys. 44 (1966) 3897.

[4] K. F. Milfeld and R. Wyatt, Phys. Rev. A 27 (1983) 72.

[5] J. H. Shirley, Phys. Rev. 138 (1965) B979.

[6] H. Sambe, Phys. Rev. A 7 (1973) 2203.

[7] M. C. Gutzwiller, Chaos in Classical and Quantum Mechanics, Springer, New York, 1990.

[8] B. Mirbach and H. J. Korsch, J. Phys. A 27 (1994) 6579.

[9] A. J. Lichtenberg and M. A. Lieberman, Regular and Stochastic Motion, Springer, New York, 1983.

[10] M. Tabor, Chaos and Integrability in Nonlinear Dynamics, John Wiley, New York, 1989.

[11] F. Bensch, H. J. Korsch, B. Mirbach, and N. Ben-Tal, J. Phys. A 25 (1992) 6761.

[12] K.-E. Thylwe and F. Bensch, J. Phys. B 27 (1994) 7474.

[13] H. R. Lewis and W. B. Riesenfeld, J. Math. Phys. 10 (1969) 1458.
[14] D. B. Monteoliva, H. J. Korsch, and J. A. Nuñez, J. Phys. A 27 (1994) 6897.

[15] R. S. Kaushal and D. Parashar, Phys. Rev. A 55 (1997) 2610.

[16] J. R. Ray, Phys. Rev. A 26 (1982) 729; 28 (1983) 2603.

[17] H. J. Korsch and H. Laurent, J. Phys. B 14 (1981) 4213; H. J. Korsch, H. Laurent, and R. Möhlenkamp, J. Phys. B 15 (1982) 1.

[18] J. A. Nuñez, F. Bensch, and H. J. Korsch, J. Phys. A 24 (1991) 2069.

[19] M. Abramowitz and I. A. Stegun, Handbook of Mathematical Functions, Dover Publications, New York, 1970.

[20] B. V. Chirikov, Phys. Rep. 52 (1979) 263.

[21] V. Croquette and C Poitou, J. Physique Lettres 42 (1981) L537.

[22] H. Frahm and H. J. Mikeska, Z. Phys. B 60 (1985) 117.

[23] G. B. Berman, O. F. Vlasova, and F. M. Israilev, Sov. Phys. JETP 66 (1987) 269.

[24] M. Toda and K. Ikeda, J. Math. Gen. 20 (1987) 3833.

[25] L. E. Reichl, The Transition to Chaos, Springer, New York, 1992.

[26] N. Moiseyev, H. J. Korsch, and B. Mirbach, Z. Phys. D 29 (1994) 125.

[27] V. Averbuckh, N. Moiseyev, B. Mirbach, and H. J. Korsch, Z. Phys. D 35 (1995) 247.

[28] L. Sirko and P. M. Koch, Appl. Phys. B 60 (1995) S195.

[29] B. Friedrich and D. R. Herschbach, Lett. to Nature $\mathbf{3 5 3}$ (1991) 412.

[30] T. Gorin, H. J. Korsch, and B. Mirbach, Chem. Phys. (1997) 147. 
TABLE I. Quasiangles $\theta_{\alpha}$ for the rigid rotor for $f=0.1$. The exact numerical values $\theta_{\alpha}^{\text {ex }}$ are compared with approximated $\theta_{\alpha}^{\mathrm{I}}$ calculated using the invariant operator $\hat{I}^{ \pm} ; \theta_{\alpha}^{\mathrm{II}}$ are the quasiangles for the corrected invariant aproximation. The column $\theta_{\alpha}^{\text {ho }}$ lists some results obtained from a harmonic oscillator approximation for low states. The horizontal line is the location of the separatrix. The states are numbered by the index $\alpha$ with increasing kinetic energy. In addition, the quantum number $n$ of the eigenstates of the invariant is given.

\begin{tabular}{cccccc|ccccc}
\hline \hline$n$ & $\alpha$ & $\theta_{\alpha}^{\text {ex }}$ & $\theta_{\alpha}^{\text {I }}$ & $\theta_{\alpha}^{\text {II }}$ & $\theta_{\alpha}^{\text {ho }}$ & $n$ & $\alpha$ & $\theta_{\alpha}^{\text {ex }}$ & $\theta_{\alpha}^{\text {I }}$ & $\theta_{\alpha}^{\text {II }}$ \\
\hline 0 & 79 & 0.8000 & 0.7011 & 0.8017 & 0.8026 & $\vdots$ & $\vdots$ & $\vdots$ & $\vdots$ & $\vdots$ \\
1 & 80 & 2.1972 & 2.0982 & 2.1987 & 2.2076 & 51 & 78 & 2.7759 & 2.6737 & 2.7742 \\
2 & 82 & -2.6967 & -2.7958 & -2.6953 & -2.6706 & 52 & 77 & -2.7469 & -2.8490 & -2.7485 \\
3 & 83 & -1.3154 & -1.4147 & -1.3142 & -1.2656 & 53 & 75 & -2.0166 & -2.1185 & -2.0180 \\
4 & 84 & 0.0576 & -0.0417 & 0.0588 & 0.1393 & 54 & 74 & -1.3385 & -1.4400 & -1.3395 \\
5 & 86 & 1.4225 & 1.3230 & 1.4235 & 1.5443 & 55 & 76 & -0.6590 & -0.7604 & -0.6599 \\
6 & 87 & 2.7792 & 2.6796 & 2.7801 & 2.9492 & 56 & 73 & -0.2083 & -0.3087 & -0.2080 \\
7 & 89 & -2.1555 & -2.2553 & -2.1548 & -1.9289 & 57 & 72 & 0.7207 & 0.6165 & 0.7170 \\
8 & 90 & -0.8155 & -0.9155 & -0.8150 & -0.5240 & 58 & 130 & 0.6133 & 0.5151 & 0.6156 \\
9 & 92 & 0.5159 & 0.4158 & 0.5163 & 0.8807 & 59 & 71 & 1.9885 & 1.8626 & 1.9631 \\
$\vdots$ & $\vdots$ & $\vdots$ & $\vdots$ & $\vdots$ & $\vdots$ & 60 & 131 & 1.9414 & 1.8626 & 1.9631 \\
25 & 118 & 1.7332 & 1.6313 & 1.7318 & & 61 & 70 & -2.8061 & -2.9352 & -2.8347 \\
26 & 120 & 2.9039 & 2.8019 & 2.9024 & & 62 & 132 & -2.8579 & -2.9352 & -2.8347 \\
27 & 122 & -2.2192 & -2.3212 & -2.2207 & & $\vdots$ & $\vdots$ & $\vdots$ & $\vdots$ & $\vdots$ \\
\hline \hline
\end{tabular}

